\title{
Signal-to-Noise Ratio Estimation Algorithm for ACM in Advanced DVB-RCS Satellite Systems
}

\author{
A. ljaz, A.B. Awoseyila and B.G. Evans \\ Centre for Communication Systems Research (CCSR), University of Surrey, \\ Guildford, GU2 7XH, Surrey, UK \\ E-mail:a.ijaz@surrey.ac.uk
}

This paper presents a signal-to-noise ratio (SNR) estimation algorithm for advanced DVB-RCS systems using adaptive coding and modulation (ACM) in the reverse link of broadband satellite systems. Due to the absence of a repetitive pilot symbol structure, SNR estimation has to be performed using the fixed symbol preamble data. Moreover, sporadic nature of data traffic on the return link causes variation in interference level from slot to slot and, therefore, the estimation has to be done within one traffic slot duration. Hence, it becomes necessary to use a combination of data-aided (DA) and decision-directed (DD) algorithms so as to make use of traffic data. A nondata-aided (NDA) estimator that was previously proposed by the authors for binary phase shift keying (BPSK) and QPSK schemes is extended to 8-PSK in a decision directed manner. This estimator shows improved performance over existing estimators. The inherent bias of DD approach at low values of SNR is reduced by using a hybrid approach, i.e. using the proposed estimator at moderate/high values of SNR and the moments-based estimator $\left(M_{2} M_{4}\right)$ at low values of SNR. Overall improved performance of the proposed hybrid estimator, in terms of accuracy and complexity, makes it an attractive choice for implementing ACM in advanced DVB-RCS systems. 


\section{Introduction}

Adaptive coding and modulation (ACM) is implemented in satellite communication systems operating at Ku band and higher frequencies in order to overcome the effect of bad channel conditions arising from rain attenuation, which can render the system economically inefficient. The resulting potential for increase in the system capacity and availability has been demonstrated in the literature [1, 2]. ACM is a type of fade mitigation technique (FMT) and the procedure involves dynamic adaptation of the modulation scheme and coding rate (i.e. ModCod) according to the prevailing channel conditions. It helps to mitigate for slow channel fading mostly caused by rain attenuation and helps to improve the link availability and provides capacity gains as compared to a non-adaptive system [3-5]. For efficient implementation of ACM, an accurate estimate of channel condition is required that is generally provided in terms of signal-to-noise ratio (SNR). Therefore, the accuracy of SNR estimation algorithms directly affects the efficiency of ACM and it is a major topic of interest for broadband satellite systems based on DVB-S2 and DVB-RCS which employ ACM.

Different SNR estimation algorithms for constant envelope signals in complex Additive White Gaussian Noise (AWGN) channel are proposed in literature [6-15]. Several data-aided (DA) and non-data-aided (NDA) estimators are compared in [13] for binary phase shift keying (BPSK) and 8-PSK signals in an AWGN channel to identify the "best" estimator in a digital receiver with the least cost. In order to assess their relevant performances, the mean square error (MSE) was used as performance metric and the absolute levels of performances were also established by comparing the simulated performances with the Cramer-Rao bound (CRB). Results show that when perfect knowledge of transmitted signal is available, i.e. DA estimation, the maximum likelihood (ML) estimator provides an efficient solution in the AWGN channel. However, NDA/DD estimators (also known as in-service estimators) are also of particular interest when training symbols are not available and/or a continuous 
estimate of SNR is required. In [13], second- and fourth- order moments based estimator (called $M_{2} M_{4}$ ) and decision directed $\mathrm{ML}$ estimators were found to be efficient in-service estimators and the choice between these two depends on the number of received symbols used for estimation, the number of samples per symbol available, the type of modulation used and the SNR range of interest.

Although various SNR estimation methods for the AWGN channel have been proposed in the literature, there is not much work done in this area from the system point of view (i.e. evaluating the estimators on a reference system) except in [16] and [17]. In [16] and [17], the performances of contemporary SNR estimation algorithms were quantified in terms of number of received symbols needed to obtain an estimate with a given error margin. Their suitability as channel quality indicators for a typical digital video broadcasting (DVB) type satellite system was analyzed by considering the various assumptions involved in the algorithms, the effect of noise due to interference and the fast fluctuations of the propagation channel during rainy conditions. It was concluded that the DA ML estimator is the best choice for the high speed forward link compliant with the DVB-S2 standard in a broadband Interactive Satellite System. This is because pilot symbols, periodically repeated within each frame in DVB-S2, enable DA estimation using the optimally efficient $M L$ algorithm to estimate SNR with an error margin of $0.2 \mathrm{~dB}$ within few miliseconds. However, this level of accuracy cannot be achieved on the return link adhering to DVB-RCS standard due to absence of a repetitive pilot symbol structure and the use of a short preamble consisting of only 48 symbols for each burst transmission. The traffic in the return link mainly consists of requests from users handling interactive applications, which is by nature sporadic with low data rate. In the DVB-RCS scheme, data are transmitted in superframes made up of frames which are themselves made of traffic slots. In the worst case, each user may transmit within one traffic slot per superframe. In the above scenario and due to slot to slot variation in interference level resulting from sporadic nature of data traffic on the return link, it is necessary to 
perform SNR estimation within one traffic slot duration [16] employing all available symbols (preamble and data) for reliable channel estimation with an acceptable error margin. Therefore, decision-directed and non-data-aided estimators are of particular interest and the performance of DDML and $M_{2} M_{4}$ estimators presented in [13] can be considered as the benchmark in this scenario for both QPSK and 8-PSK modulations.

In [9], Beaulieu et al. proposed four different SNR estimators for QPSK modulation and the estimator denoted as $\hat{\theta}_{2}$ was shown to exhibit the best performance among the four techniques. This estimator can be used only for QPSK-like signal whose baseband signal symbol constellation has four symbol points that form a square centred at the origin of the signal space. The estimator uses the difference between the in-phase and quadrature components of the received signal to estimate noise power and the second order moment of the signal was used to estimate the signal power. Another NDA estimator for constant envelope signals was proposed in [10] for M-PSK signals. The estimator takes $M^{\text {th }}$ ( $M$ denotes the size of the constellation) power of the received signal to remove phase modulation and estimates the signal power using the ML estimation principle. The performance of this estimator degrades due to the noise penalty introduced by the $M^{\text {th }}$ power process as the constellation order increases.

We propose a NDA/DD SNR estimator which can be used for full scale ACM, employing QPSK and 8-PSK modulation schemes with different code rates, in future DVB-RCS systems providing interactive broadband services to fixed terminals. The authors have already published an NDA SNR estimation algorithm for BPSK and QPSK modulation schemes in the literature [18], which improves on the existing methods in terms of complexity and accuracy. Therefore, in this paper we propose an extension of this algorithm to 8-PSK in a decision-directed manner. Section 2 presents the signal model and formulates the SNR estimation problem while section 
3 reviews the SNR estimation algorithm proposed in [18] for BPSK and QPSK modulation schemes, followed by an extension of this algorithm to make it applicable to 8-PSK modulation. Simulation results are presented in section 4 to verify the accuracy of the proposed scheme in DVB-RCS compliant systems. Finally, the conclusion is provided in section 5 .

\section{Signal Model and SNR Estimation}

Since we tend to address the issue of SNR estimation on the levels of slot in DVBRCS system providing broadband services to fixed terminals, the estimation duration is in the order of few milliseconds with negligible variation in scintillation level and rain fade [16]. Therefore, signal fading can be assumed to remain constant and the link can be modeled as an AWGN channel. Furthermore, a full scale ACM with QPSK and 8-PSK with different coding rates is envisaged in future DVB-RCS systems [19]. Hence in this system, signal model and SNR estimation problem can be formulated as follows.

Let $S_{I_{k}}$ and $S_{Q_{k}}, k=1,2, \ldots, L$, be the in-phase and quadrature components of a symbol belonging to a phase shift keying (PSK) constellation transmitted over an AWGN channel. The signal components are assumed to be independent and identically distributed (i.i.d.) discrete random variables. Assuming one complex sample is taken for each of the $L$ symbols transmitted and that carrier synchronisation exists, the $k^{\text {th }}$ received signal, $Z_{k}=X_{k}+j Y_{k}$, can be described as:

$$
\begin{gathered}
X_{k}=S_{I_{k}}+n_{I_{k}} \\
Y_{k}=S_{Q_{k}}+n_{Q_{k}}
\end{gathered}
$$


where $X_{k}$ and $Y_{k}$ represent the in-phase and quadrature components of $Z_{k}$ respectively, while $n_{I_{k}}$ and $n_{Q_{k}}$ represent the in-phase and quadrature components of noise which are taken to be zero-mean i.i.d. Gaussian random variables, with variances $\sigma_{I}^{2}=\sigma_{Q}^{2}=\sigma^{2}$, respectively. The SNR, $\rho$, of the received signal is given by:

$$
\rho=\frac{E\left\{S_{I_{k}}^{2}+S_{Q_{k}}^{2}\right\}}{E\left\{n_{I_{k}}^{2}+n_{Q_{k}}^{2}\right\}}=\frac{S}{N}
$$

We now review the two state-of-the-art DD and NDA estimators that are suitable for the system under consideration, i.e., DDML and $M_{2} M_{4}$ algorithms. DDML is a decision directed algorithm based on maximum likelihood estimation theory [20] and, as given in [13], is reproduced here for completeness:

$$
\begin{gathered}
\hat{S}_{D D M L}=\left[\frac{\frac{1}{L} \sum_{k=1}^{L}\left(X_{k} \hat{S}_{I_{k}}+Y_{k} \hat{S}_{Q_{k}}\right)}{\frac{1}{L} \sum_{k=1}^{L}\left(\hat{S}_{I_{k}}{ }^{2}+\hat{S}_{Q_{k}}{ }^{2}\right)}\right]^{2} \\
\hat{N}_{D D M L}=\frac{1}{L} \sum_{k=1}^{L}\left(X_{k}{ }^{2}+Y_{k}{ }^{2}\right)-\hat{S}_{D D M L} \frac{1}{L} \sum_{k=1}^{L}\left(\hat{S}_{I_{k}}{ }^{2}+\hat{S}_{Q_{k}}{ }^{2}\right)
\end{gathered}
$$

where $\hat{S}_{D D M L}$ and $\hat{N}_{D D M L}$ are the respective estimates of signal and noise power and $\hat{S}_{I k}$ and $\hat{S}_{Q k}$ are the estimates of transmitted signal's real and imaginary components. $M_{2} M_{4}$ algorithm [13] estimates signal and noise power based on second- and fourthorder moments of the received signal as given below:

$$
\begin{gathered}
\hat{S}_{M_{2} M_{4}}=\sqrt{2 M_{2}^{2}-M_{4}} \\
\hat{N}_{M_{2} M_{4}}=M_{2}-\sqrt{2 M_{2}^{2}-M_{4}}
\end{gathered}
$$


An estimate of $M_{2}$ and $M_{4}$ is used in (6) and (7), determined from the received signal's samples as:

$$
\begin{aligned}
& \hat{M}_{2}=\frac{1}{L}\left[\sum_{k=1}^{L}\left|X_{k}\right|^{2}+\sum_{k=1}^{L}\left|Y_{k}\right|^{2}\right] \\
& \hat{M}_{4}=\frac{1}{L}\left[\sum_{k=1}^{L}\left|X_{k}\right|^{4}+\sum_{k=1}^{L}\left|Y_{k}\right|^{4}\right]
\end{aligned}
$$

\section{Proposed Estimator}

\subsection{Estimator for BPSK and QPSK}

An NDA SNR estimator was proposed for BPSK and QPSK signals in [18] based on an observation that absolute values of the in-phase and quadrature components of the received signal have a close relationship with signal power, since these components have a constant amplitude in the transmit signal. Consequently, signal power estimate from the received signal samples was proposed as follows:

$$
\hat{S}=\frac{1}{L^{2}}\left[\left(\sum_{k=1}^{L}\left|X_{k}\right|\right)^{2}+\left(\sum_{k=1}^{L}\left|Y_{k}\right|\right)^{2}\right]
$$

The SNR estimate is given as:

$$
\hat{\rho}=\frac{\hat{S}}{\hat{M}_{2}-\hat{S}}
$$

where $\hat{M}_{2}$ is an estimate of total received power determined from (8).

\subsection{Proposed Estimator for 8-PSK}

The observed property of constant amplitude of the in-phase and quadrature components is not valid for 8-PSK signals, which means that the estimator in (10) is not directly applicable. However, as shown in Fig. 1, it can be observed that the 8- 
PSK constellation consists of one QPSK constellation (marked with $\oplus$ ) and two orthogonal BPSK constellations (marked with $\ominus$ and $Q$ ).

For the transmitted symbols belonging to one of these subsets $n$, where $n=1,2,3$, we observe that the absolute values of the amplitudes of in-phase and quadrature components remain constant. Therefore, we can still use the estimator in (10) to independently estimate the average signal power in each subset, and then use a weighted average of the three estimates to determine the average signal power of the constellation. This proposed extension to 8-PSK is analysed accordingly in (12) (27).

The absolute values of the in-phase and quadrature components of the received signal as presented in (1) and (2) are given as follows:

$$
\begin{gathered}
\left|X_{k}\right|=\left|S_{I_{k}}+W_{I_{k}}\right| \\
\left|Y_{k}\right|=\left|S_{Q_{k}}+W_{Q_{k}}\right|
\end{gathered}
$$

Assuming that $\left|W_{k}\right|<\left|S_{k}\right|$ (which is usually valid at moderate/high SNR):

$$
\begin{gathered}
\left|X_{k}\right|=\left|S_{I_{k}}\right|+W_{I_{k}} \quad\left(X_{k}>0\right) \\
\left|X_{k}\right|=\left|S_{I_{k}}\right|-W_{I_{k}} \quad\left(X_{k}<0\right) \\
\left|Y_{k}\right|=\left|S_{Q_{k}}\right|+W_{Q_{k}} \quad\left(Y_{k}>0\right) \\
\left|Y_{k}\right|=\left|S_{Q_{k}}\right|-W_{Q_{k}} \quad\left(Y_{k}<0\right)
\end{gathered}
$$

For the signal belonging to a specific subset $n$ from the 8-PSK constellation, we can replace subscript $k$ by the subscripts $n$ and $j$. It should be noted that $j=1,2, \ldots, L_{n}$ for 
for the $\mathrm{n}^{\text {th }}$ subset and the total number of received samples is $\sum_{n=1}^{3} L_{n}=L$. Using (14) (17) and given that noise components are i.i.d zero-mean Gaussian random variables, for subset 1 , we observe that the mean of absolute values of amplitude of the received signal samples are as follows:

$$
\begin{gathered}
E\left\{\left|X_{1, j}\right|\right\}=E\left\{\left|S_{I_{1, j}}\right|\right\} \pm E\left\{W_{I_{1, j}}\right\} \\
=E\left\{\left|S_{I_{1, j}}\right|\right\}=a \\
E\left\{\left|Y_{1, j}\right|\right\}=E\left\{\left|S_{Q_{1, j}}\right|\right\} \pm E\left\{W_{Q_{1, j}}\right\}=0
\end{gathered}
$$

Similarly, for subsets 2 and 3 , it is observed that:

$$
\begin{aligned}
E\left\{\left|X_{2, j}\right|\right\} & =E\left\{\left|S_{I_{2, j}}\right|\right\} \pm E\left\{W_{I_{2, j}}\right\} \\
& =E\left\{\left|S_{I_{2, j}}\right|\right\}=a / \sqrt{2} \\
E\left\{\left|Y_{2, j}\right|\right\}= & E\left\{\left|S_{Q_{2, j}}\right|\right\} \pm E\left\{W_{Q_{2, j}}\right\} \\
= & E\left\{\left|S_{Q_{2, j}}\right|\right\}=a / \sqrt{2} \\
E\left\{\left|X_{3, j}\right|\right\} & =E\left\{\left|S_{I_{3, j}}\right|\right\} \pm E\left\{W_{I_{3, j}}\right\}=0 \\
E\left\{\left|Y_{3, j}\right|\right\}= & E\left\{\left|S_{Q_{3, j}}\right|\right\} \pm E\left\{W_{Q_{3, j}}\right\} \\
& =E\left\{\left|S_{Q_{3, j}}\right|\right\}=a
\end{aligned}
$$

From (18) - (23), it can be seen that the mean of absolute values of in-phase and quadrature components of the received signal yields the amplitude of in-phase and quadrature components of the transmitted signal respectively, in the three subsets. 
Therefore, in order to estimate signal power and signal-to-noise-ratio from the absolute values of the received signal components, the proposed approach is to partition the received signal's samples into three subsets based on their phase values. Then the estimator in [18] is applied to each of these subsets to find the respective average power. Finally, an estimate of average received power is obtained as sum of the average power of three subsets according to the estimated probability of the respective subset. However, unlike BPSK and QPSK, the estimator is no longer purely NDA for 8-PSK. The proposed estimator is decision directed because the three subgroups in 8-PSK constellation are determined by hard symbol decisions in the presence of noise. Consequently, the accuracy of the proposed estimator depends on the accuracy of symbols decisions, which in turn depends on the SNR. The proposed algorithm to estimate signal power for 8-PSK is presented in Fig. 2 and can be summarized as follows:

- Iteration: $k=1,2, \ldots, L$

1. Find the angle of the $k^{\text {th }}$ received sample, i.e. find $\theta_{k}=\angle Z_{k}$.

2. Decide the $n=3$ subsets of $Z_{k}$ based on $\theta_{k}$, i.e. $\theta_{k} \Rightarrow Z_{n, j ; j=1,2, \ldots, L_{n}}$ If, $-\frac{\pi}{8}<\theta_{k}<\frac{\pi}{8} \| \frac{7 \pi}{8}<\theta_{k}<-\frac{7 \pi}{8}$, then $n=1$ Else If, $\frac{3 \pi}{8}<\theta_{k}<\frac{5 \pi}{8} \|-\frac{3 \pi}{8}<\theta_{k}<-\frac{5 \pi}{8}$, then $n=3$ Else, $n=2$

3. Calculate the absolute value of in-phase and quadrature components of the received sample based on its subset, i.e. $\left|X_{n, j ; n \neq 3}\right|=\left|\operatorname{real}\left\{Z_{n, j ; n \neq 3}\right\}\right|$ and $\left|Y_{n, j ; n \neq 1}\right|=\left|\operatorname{imag}\left\{Z_{n, j ; n \neq 1}\right\}\right|$.

- Find the average signal power of the received signal as: 


$$
\hat{S}_{\text {proposed }}=\frac{1}{L} \sum_{n=1}^{3} \hat{S}_{n} L_{n}
$$

where $L_{n}$ is the number of samples in the $n^{\text {th }}$ subset and $\hat{S}_{n}$ is an estimate of average power of the $n^{\text {th }}$ subset. $\hat{S}_{n}$ is determined for the three subsets as follows:

$$
\begin{aligned}
& \hat{S}_{1}=\frac{1}{L_{1}^{2}}\left[\left(\sum_{j=1}^{L_{1}}\left|X_{1, j}\right|\right)^{2}\right] \\
& \hat{S}_{2}=\frac{1}{L_{2}^{2}}\left[\left(\sum_{j=1}^{L_{2}}\left|X_{2, j}\right|\right)^{2}+\left(\sum_{j=1}^{L_{2}}\left|Y_{2, j}\right|\right)^{2}\right] \\
& \hat{S}_{3}=\frac{1}{L_{3}^{2}}\left[\left(\sum_{j=1}^{L_{3}}\left|Y_{3, j}\right|\right)^{2}\right]
\end{aligned}
$$

- Finally, SNR can be estimated using (8), (11) and (24).

\section{$4 \quad$ Simulation Results}

Now we analyse the performance of the proposed algorithm to identify its suitability as a channel quality indicator in a DVB-RCS system. Our aim is to quantify the performance of the proposed algorithm when SNR estimation is performed in duration of one slot only. We also find the error margin $\delta$ such that the estimation error is less than or equal to $\delta$ for $99 \%$ of the trials.

Assuming single MPEG Transport Stream (TS) packet (188 bytes) burst transmission for each assigned slot to a user and a preamble consisting of 48 symbols, we analyse the performance of the proposed estimator in a DVB-RCS system. First, we investigate the performance of the estimator for QPSK modulation. A total number of 100,000 iterations were performed in complex AWGN channel for an SNR range starting from 4.9dB (the lowest operating threshold in DVB-RCS network for a target bit error rate of $\left.10^{-5}[19]\right)$ to $19.9 \mathrm{~dB}$. 
In order to assess the absolute performance of the estimator for QPSK modulation, the normalized mean square error (NMSE) [13] of the estimator is shown in Fig. 3 along with the data aided Cramer-Rao bound $\left(\mathrm{CRB}_{\mathrm{DA}}\right)$ [13]. NMSE and $\mathrm{CRB}_{\mathrm{DA}}$ are evaluated as follows:

$$
\begin{gathered}
N M S E\{\hat{\rho}\}=\frac{1}{N_{t}} \sum_{i=1}^{N_{t}}\left(\frac{\hat{\rho}_{i}-\rho}{\rho}\right)^{2} \\
C R B_{D A}=\frac{1}{L}\left(1+\frac{2}{\rho}\right)
\end{gathered}
$$

where $N_{t}$ is the total number of simulation trials, $\hat{\rho}_{i}$ is the estimated SNR at $i^{\text {th }}$ trial and $\rho$ is the actual value of SNR. This figure also shows comparison with $M_{2} M_{4}$ estimator, DDML algorithm, the estimator $\hat{\theta}_{2}$ [9] (labelled as $\theta_{2}$ in the results) and the NDA estimator in [10] (referred to as the $M^{\text {th }}$ power ML estimator from now onwards).

It can be seen from the figure that the $M^{\text {th }}$ power $M L$ estimator has the highest NMSE as compared to the other estimators. It can also be observed from this comparison that the proposed NDA estimator is biased at low SNR values, whereas, its accuracy increases with SNR and the NMSE approaches the CRB at approximately 9dB. On the other hand, the $M_{2} M_{4}$ estimator does not approach CRB even at high values of SNR although it is less biased than the proposed estimator below $6.5 \mathrm{~dB}$. The estimator $\theta_{2}$ shows almost similar performance to the $M_{2} M_{4}$ estimator for SNR higher than $8 \mathrm{~dB}$ whereas for low SNR values, its performance is worse than the $M_{2} M_{4}$ estimator. It is also observed that the accuracy of DDML estimator is similar to the proposed estimator.

Now let us observe the accuracy of the proposed estimator for 8-PSK modulation in complex AWGN for an SNR range starting from $9.6 \mathrm{~dB}$ (the lowest operating threshold for 8PSK for a target bit error rate of $10^{-5}$, [19]) to $24.6 \mathrm{~dB}$. In this case, 
without any knowledge of transmitted symbols, only decision directed version of the proposed algorithm is applicable and the results are shown in Fig. 4. Due to the poor performance of $\mathrm{M}^{\text {th }}$ power $\mathrm{ML}$ estimator, it is now not considered for comparison. The estimator $\theta_{2}$ is only applicable to QPSK modulations; therefore, Fig. 4 represents the NMSE performance of the proposed estimator compared to $M_{2} M_{4}$ and DDML algorithms only. It can be observed from Fig. 4 that $M_{2} M_{4}$ has reduced bias than DDML and the proposed estimator below $12.5 \mathrm{~dB}$ but the later two estimators approach CRB at approximately $14.6 \mathrm{~dB}$, whereas, $M_{2} M_{4}$ does not attain this accuracy.

It is observed from the simulation results that the proposed estimator performs better than the $M_{2} M_{4}$ estimator and shows significant improvement in accuracy especially at moderate/high SNR for both QPSK and 8-PSK schemes. The accuracy of the proposed estimator is found to be similar to that of DDML estimator. However, overall complexity of the proposed estimator is less than both estimators as can be seen from Table 1. This table shows the number of real additions and multiplications required to estimate signal power using the three estimators assuming equiprobable distribution of transmitted 8-PSK constellation. For $M_{2} M_{4}$ estimator, the number of computations required to calculate $M_{2}$ is not taken into account, since, it is required to estimate SNR in all the three algorithms.

For a single MPEG TS packet carrying 8-PSK modulated symbols (i.e. 501 symbols), the proposed estimator requires only 11 real multiplications (it is independent of the number of estimation symbols ' $L$ ') and 751 additions, whereas DDML requires 1001 multiplications and 1004 additions and $M_{2} M_{4}$ requires 1507 multiplications and 501 additions (both having a multiplication complexity which increases with $L$ ).

Now that we have analyzed the performance of this estimator in DVB-RCS network and compared it with the state-of-the-art DD and NDA estimators, the proposed 
estimator is shown to be a better choice than DDML estimator as both have the same performance in terms of accuracy but the proposed algorithm is less complex. In the low SNR region, the condition $\left|W_{k}\right|<\left|S_{k}\right|$ is not satisfied which implies that (14) - (23) do not hold true and, therefore, the proposed estimator shows increased bias. Although the $M_{2} M_{4}$ estimator is more complex and is less accurate, at moderate/high SNR, it has lower NMSE than the proposed estimator at low SNR values. Therefore, it is further proposed to use a hybrid algorithm that first estimates SNR according to the less complex proposed algorithm. Since the proposed estimator is accurate at high values of SNR, therefore if the estimated SNR is above a certain predetermined threshold, the estimated value is accepted as a reliable measure of the SNR. However, if the estimated value is below the threshold, then the SNR is estimated according to the $M_{2} M_{4}$ estimator to obtain more accurate estimate of SNR. The threshold levels are selected as $6.5 \mathrm{~dB}$ and $12.5 \mathrm{~dB}$ for QPSK and 8PSK, respectively, from Fig. 3 and 4. The resulting NMSE that can be achieved using the hybrid estimator is represented in Fig. 5 and 6 . It can be seen from these curves that in the transition region, where the estimator switches from proposed amplitude based algorithm to the $M_{2} M_{4}$ estimator, there is higher variance than can be achieved by the more accurate $M_{2} M_{4}$ algorithm for SNR below threshold level. This is due to the error margin associated with the proposed approach and, therefore, it is important to adjust the threshold levels to compensate for the variance in estimation. As shown in [19], the ModCod selections for ACM in DVB-RCS can vary in steps of $0.2 \mathrm{~dB}$ to $1 \mathrm{~dB}$. This implies a high level of required accuracy. Error margin is yet not specified for future DVB-RCS systems employing full scale ACM but the best accuracies that can be obtained using state-of-the-art techniques such as the proposed estimator will define possible threshold levels. Extensive computer simulations were performed to find the error margin attained by $M_{2} M_{4}$ and the 
proposed estimator and the results have indicated that the $M_{2} M_{4}$ estimator attains an error margin of $1 \mathrm{~dB}$ whereas the proposed estimator achieves a better error margin of $0.5 \mathrm{~dB}$. These results were obtained assuming a worst case scenario that only one traffic slot is allotted to a user per superframe, and single MPEG TS packet is transmitted per slot. Accuracy and error margin will be further improved when more packets are available within a burst. When estimation is performed on the level of slots, $0.5 \mathrm{~dB}$ margin is accounted for and the threshold levels for switching are set to $7 \mathrm{~dB}$ and $13 \mathrm{~dB}$ for QPSK and 8-PSK, respectively, with the simulation results presented in Fig. 7 and 8.

\section{Conclusions}

We have proposed a reduced complexity and improved accuracy NDA/DD SNR estimator for use in future DVB-RCS systems employing ACM. The estimator makes use of only amplitude and phase values of the received signal in its estimation/decision process such as to achieve significant improvement in performance (i.e. lower complexity and greater accuracy) than the existing estimators for both QPSK and 8-PSK. In order to reduce the bias at low values of SNR, a hybrid approach is further proposed using the proposed method to estimate SNR and if the estimated SNR falls below a certain predetermined threshold, then $M_{2} M_{4}$ estimator is used. The proposed hybrid approach shows promising results for the operating SNR regions of ACM in DVB-RCS, as analysed in the literature, even in the worst case scenario of a single MPEG TS packet for each burst transmission.

\section{Acknowledgement}

This work was supported in part by the European SatNEx project.

\section{$7 \quad$ References}


[1] Boussemart, V., and Brandt, H.: 'Performance Assessment Using ACM in DVBS2/Advanced DVB-RCS Satellite Systems', $27^{\text {th }}$ AIAA Int. Commun. Satellite Systems Conf., 2009, pp. 212-220.

[2] Gardikis, G., Zotos, N., and Kourtis, A.: 'Satellite Media Broadcasting with Adaptive Coding and Modulation', Int. J. of Digital Multimedia Broadcasting, 09, 2009.

[3] Caire, G., and Kumar, K.R.: 'Information Theoretic Foundations of Adaptive Coded Modulation', Proc. of the IEEE , Dec. 2007, 95, (12), pp. 2274-2298.

[4] Cioni, S., De Gaudenzi, R., and Rinaldo, R.: 'Adaptive Coding and Modulation for the Forward Link of Broadband Satellite Networks', Proc. IEEE Global Telecommun. Conf., Dec. 2003, 6, pp. 3311-3315.

[5] Cioni, S., De Gaudenzi, R., and Rinaldo, R.: 'Adaptive Coding and Modulation for the Reverse Link of Broadband Satellite Networks', Proc. IEEE Global Telecommun. Conf., 2004, 2, pp. 1101-1105.

[6] Kerr, R. B.: 'On Signal and Noise Level Estimation in a Coherent PCM Channel', IEEE Trans. Aerosp. Electron. Syst., Jul. 1966, AES-2, pp. 450-454.

[7] Gagliarddi, R. M., and Thomas, C. M.: 'PCM Data Reliability Monitoring Through Estimation of Signal-to-Noise Ratio', IEEE Trans. Commun., Jun. 1968, Com-16, pp. 479-486.

[8] Benedict, T. R., and Soong, T. T.: 'The Joint Estimation of Signal and Noise from the Sum Envelope', IEEE Trans. Inform. Theory, Jul. 1967, IT-13, pp. 447454.

[9] Beaulieu, N. C., Toms, A. S., and Pauluzzi, D. R.: 'Comparison of Four SNR Estimators for QPSK Modulation', IEEE Commun. Lett., 2000, 4, (2), pp. 43-45.

[10] Xu, H., and Zheng, H.: 'The Simple SNR Estimation algorithm for MPSK Signals', Proc. Int. Conf. Signal Process. (ICSP), Aug. - Sep. 2004, 2, pp. 17811785.

[11] Ren, G., Chang, Y., and Zhang, H.: 'A new SNR's Estimator for QPSK Modulations in an AWGN Channel', IEEE Trans. Circuits and Systems-II: Express Briefs, Jun. 2005, 52, (2), pp. 336-338.

[12] Bakkali, M., Stephenne, A., and Affes, S.: 'Iterative SNR Estimation for MPSK Modulation over AWGN Channels,' Proc. IEEE $64^{\text {th }}$ Veh. Tech. Conf. (VTC), Sep. 2006, pp. 1-5.

[13] Pauluzzi, D. R., and Beaulieu, N. C.: 'A Comparison of SNR Estimation Techniques for the AWGN Channel', IEEE Trans. Commun., Oct. 2000, 48, (10), pp. 1681-1691.

[14] Trachanas, I., Fliege, N. J.: 'A novel phase based SNR estimation method for constant modulus constellations', $3^{\text {rd }}$ Int. Symp. on Commun., Control and Signal Process., 2008, (ISCCSP 2008)., Mar. 2008, pp. 1179-1183. 
[15] Pei-jun, Y., and Jian-ping, A.: 'SNR estimation for constant envelope signals in AWGN channel', Int. Conf. Wireless Commun., Networking and Mobile Computing, Sep. 2005, pp. 525-528.

[16] Aroumont, A., Alamanac, A.-B., Castanet, L., and Bousquet, M.: 'SNR Estimation Algorithms for Channel Quality Detection in Ka/EHF Band Satellite Systems Employing Fade Mitigation Techniques', First European Conf. on Antennas and Propagation, EuCAP 2006, Nov. 2006, pp. 1-6.

[17] Aroumont, A., Castanet, L., and Bousquet, M.: 'A Study on SNR Estimation Algorithms for Channel State Estimation in Communication Satellite Systems Employing Fade Mitigation Techniques', Space Commun., Oct. 2009, 22, (1), pp. 31-40.

[18] Ijaz, A., Awoseyila, A. B, and Evans, B. G.: 'Improved SNR Estimation for BPSK and QPSK Signals', IET Electron. Lett., Jul. 2009, 45, (16), pp. 858- 859.

[19] Smolinkar, M., Aroumont, A., Mohorcic, M., Javornik, T., Castanet, L.: 'On Tranmission Modes Subset Selection in DVB-S2/RCS Satellite Systems', IEEE Int. Workshop on Satellite and Space Commun., Oct. 2008, pp. 263-267.

[20] Kay, S. M.: 'Fundamentals of Statistical Signal Processing: Estimation Theory', (Prentice Hall PTR., Englewood Cliffs, NJ, 1993) 
FigurelTable captions:

Fig. 1 Three subsets in 8-PSK constellation

Fig. 2 Flow chart to estimate signal power for 8-PSK

Fig. 3 NMSE of estimated SNR for QPSK

Fig. 4 NMSE of estimated SNR for 8-PSK

Fig. 5 NMSE of hybrid estimator for QPSK

Fig. 6 NMSE of hybrid estimator for 8-PSK

Fig. 7 NMSE of hybrid estimator with adjusted threshold for QPSK

Fig. 8 NMSE of hybrid estimator with adjusted threshold for 8-PSK

Table 1 Complexity Comparison of the Estimators 
Figure 1

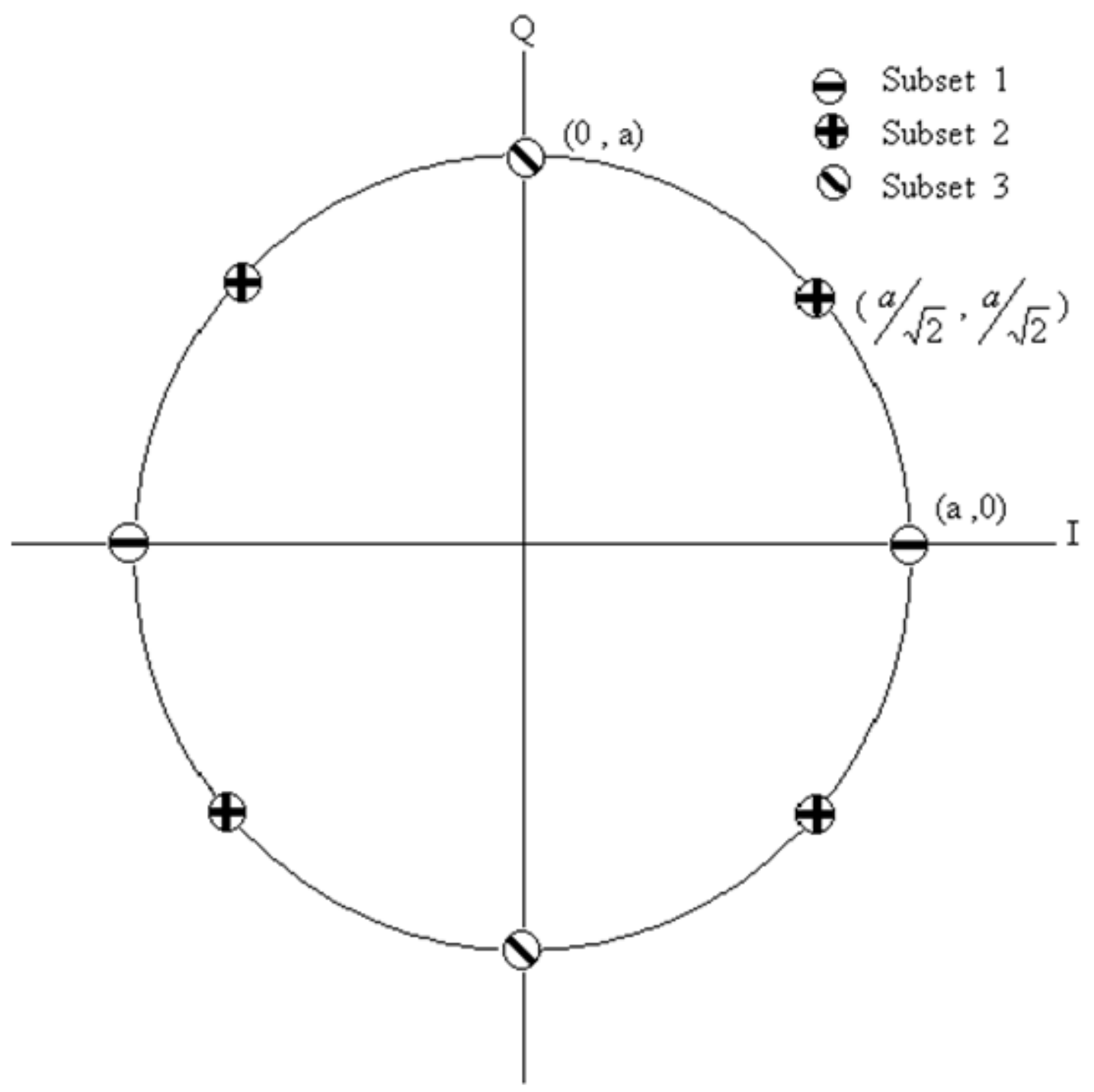


Figure 2

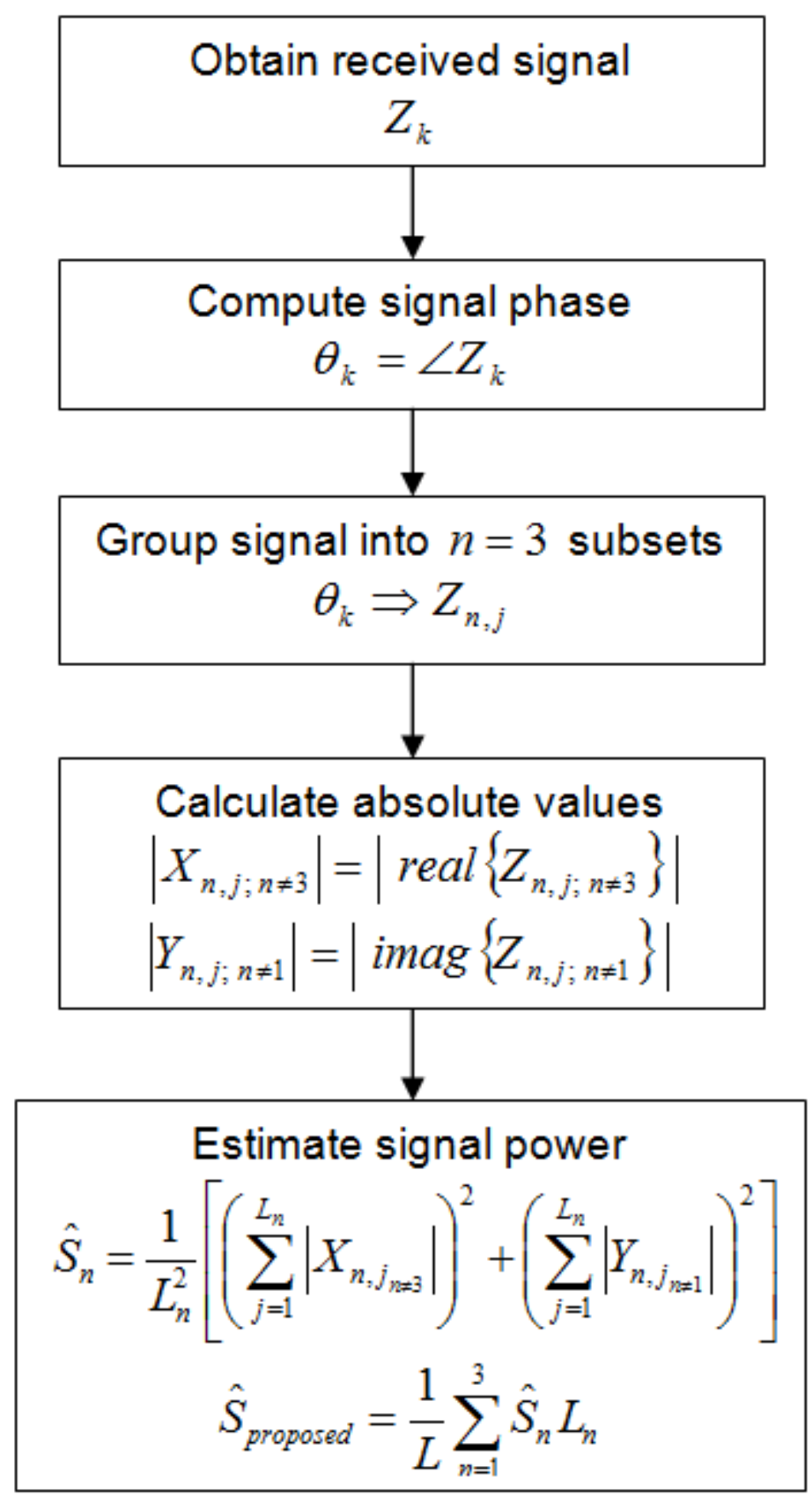


Figure 3

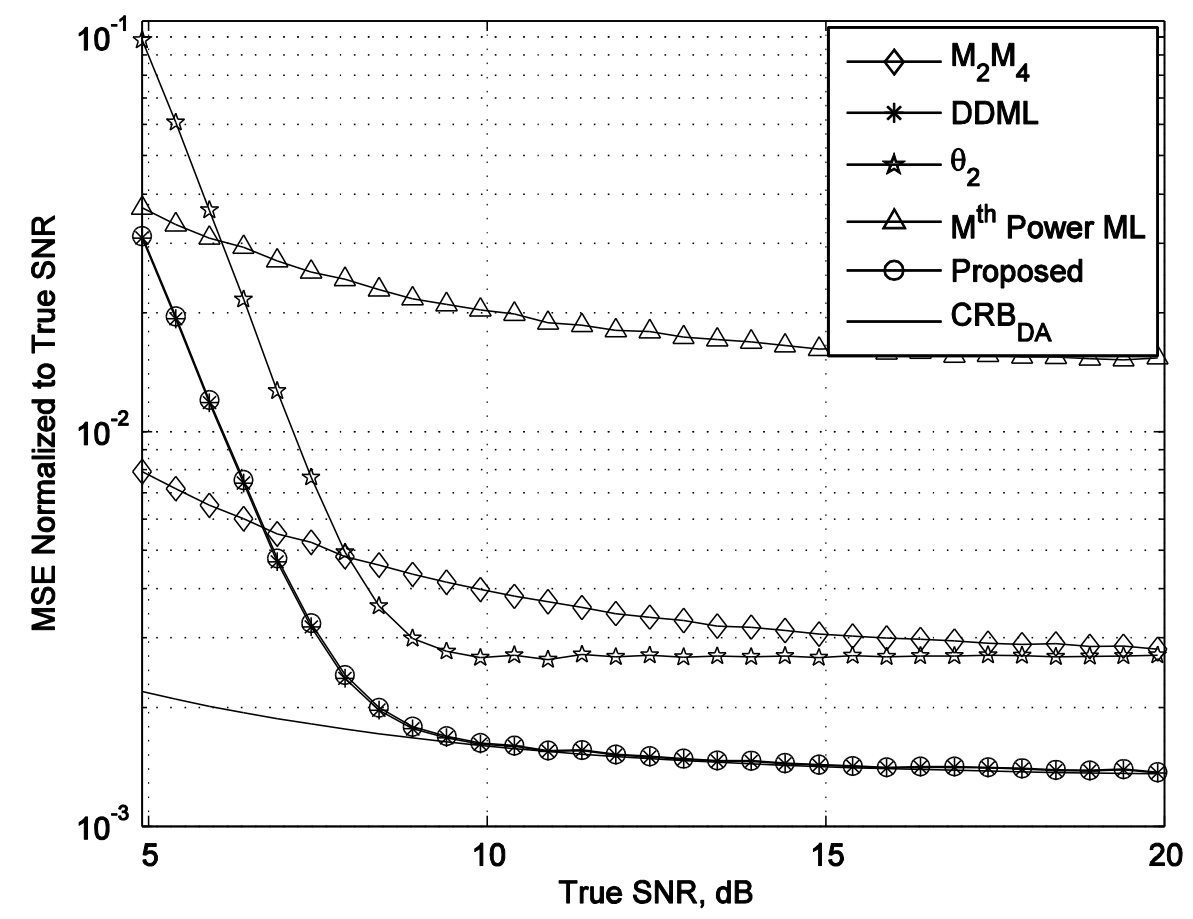


Figure 4

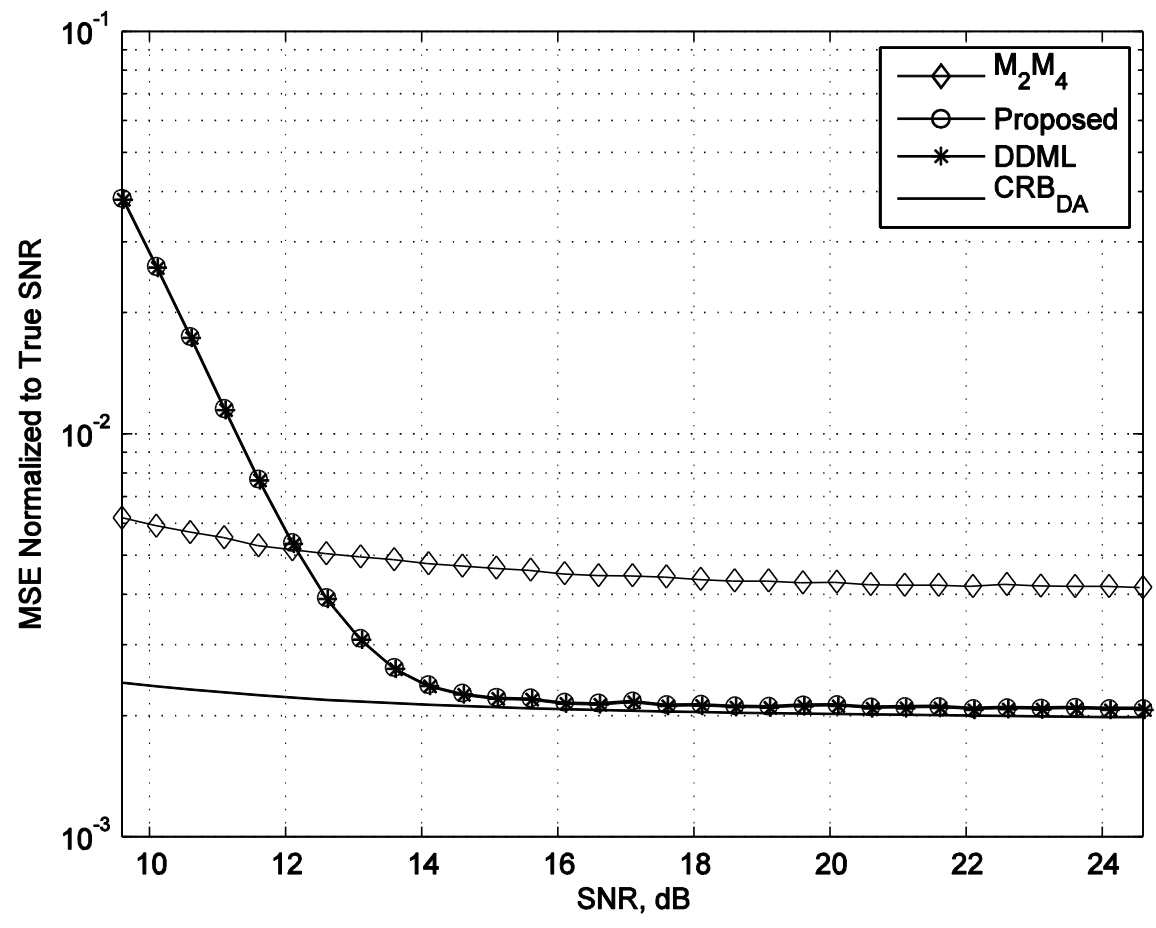


Figure 5

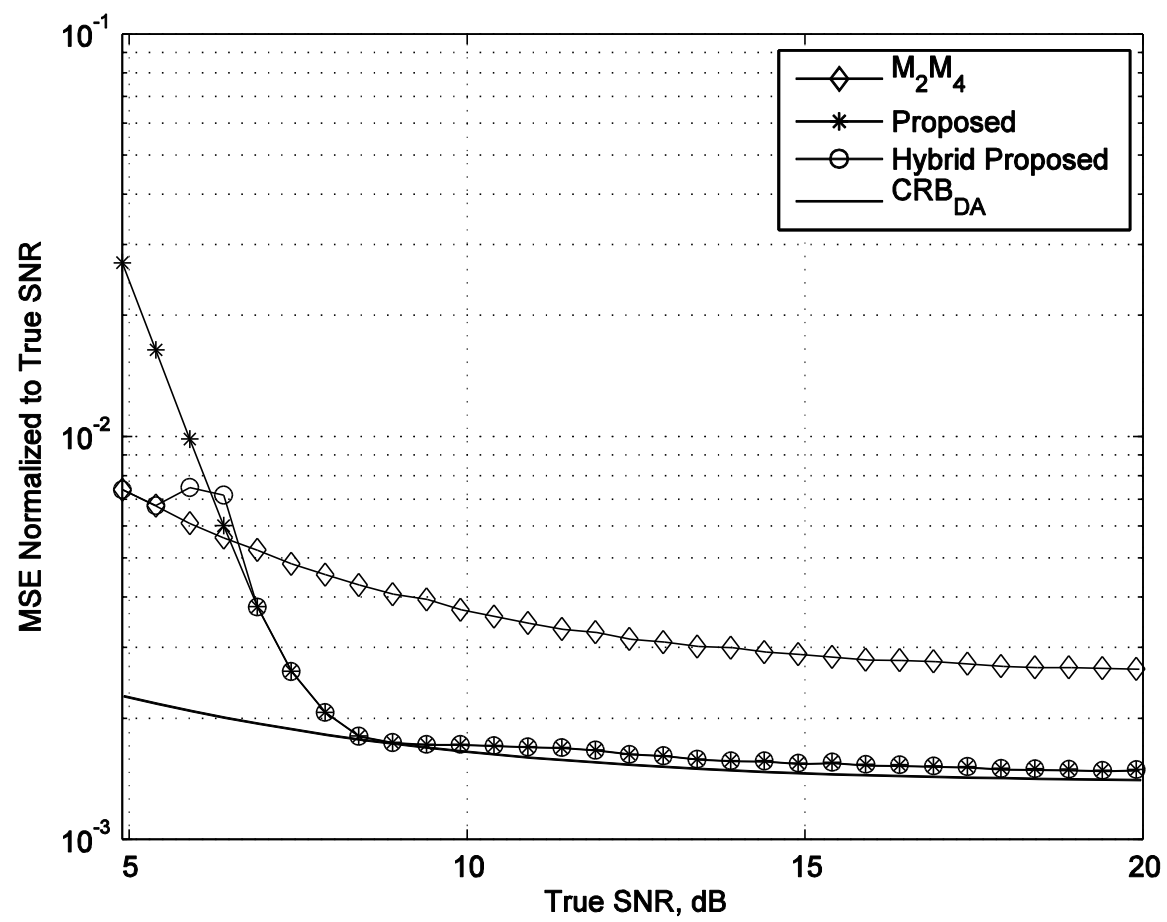


Figure 6

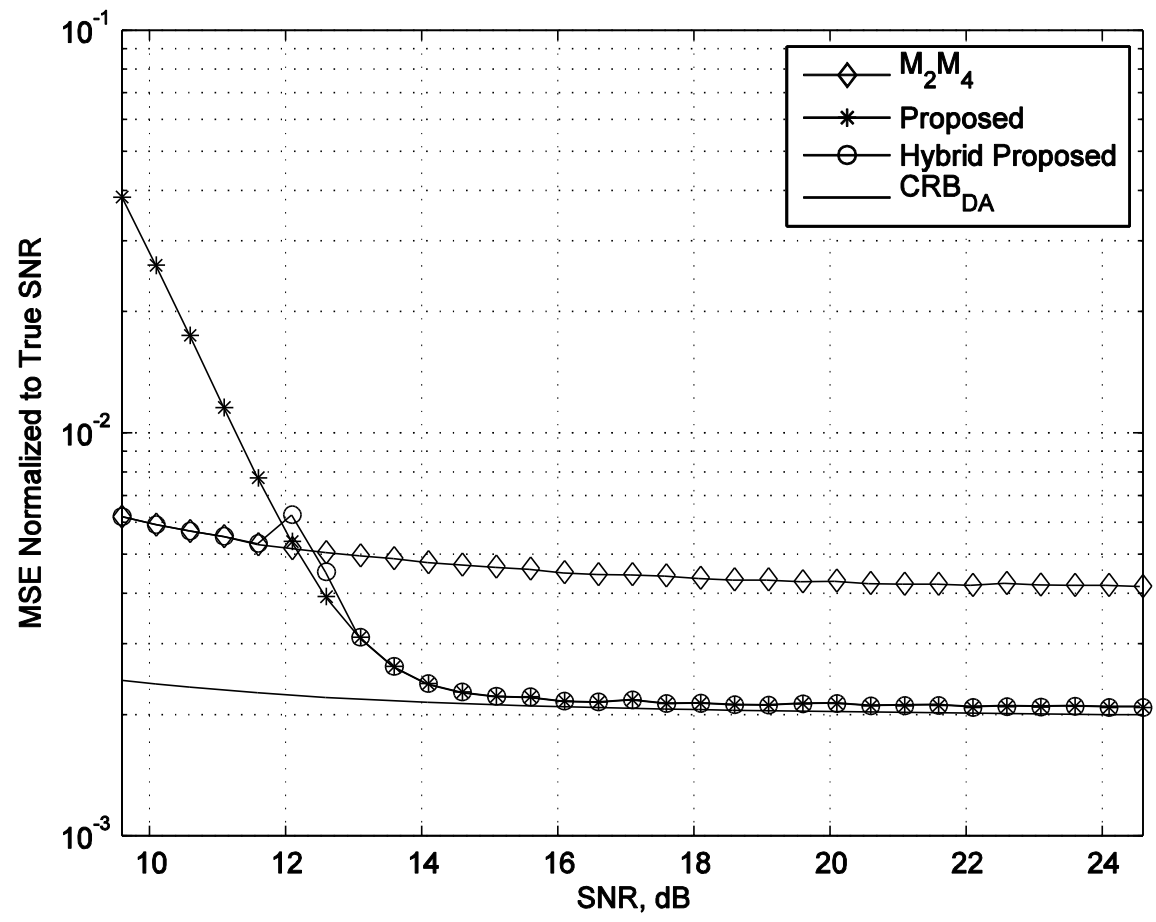


Figure 7

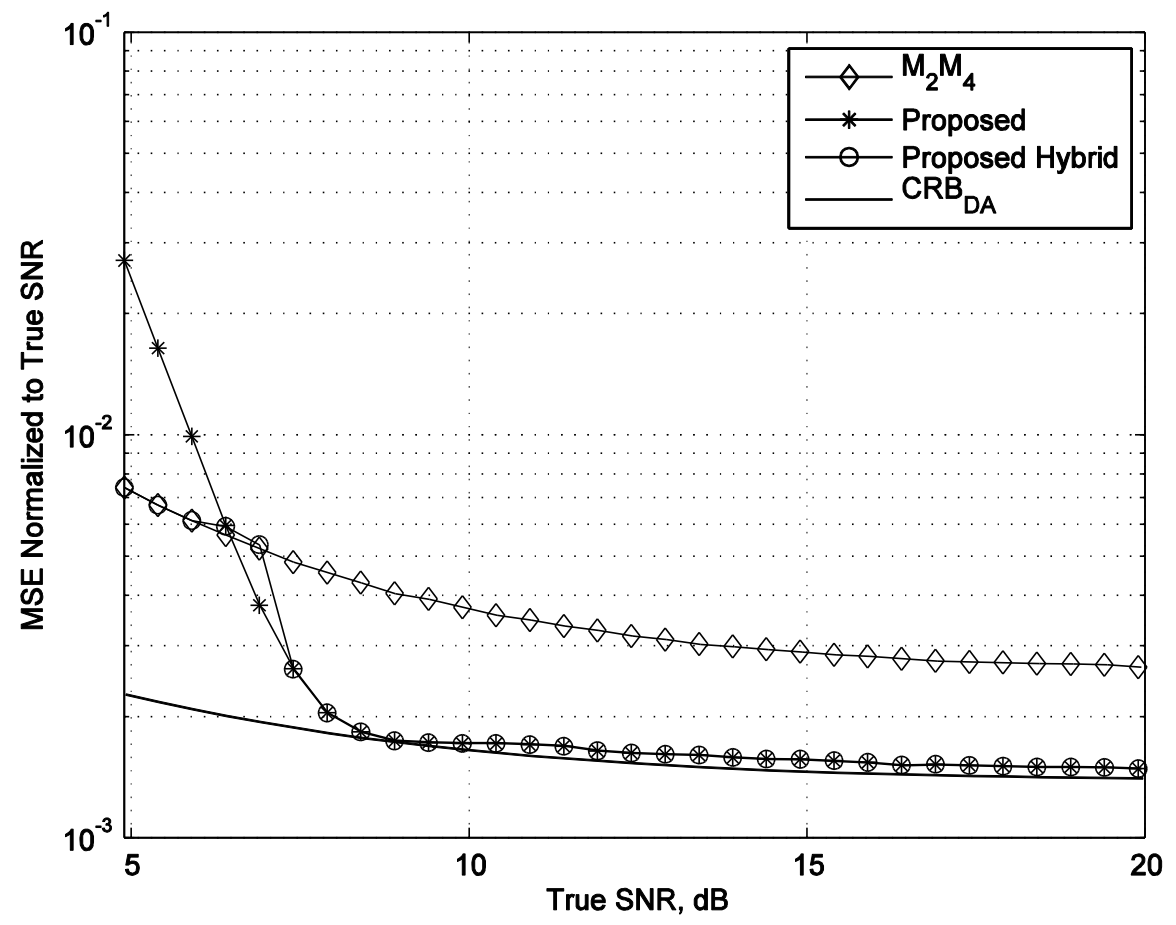


Figure 8

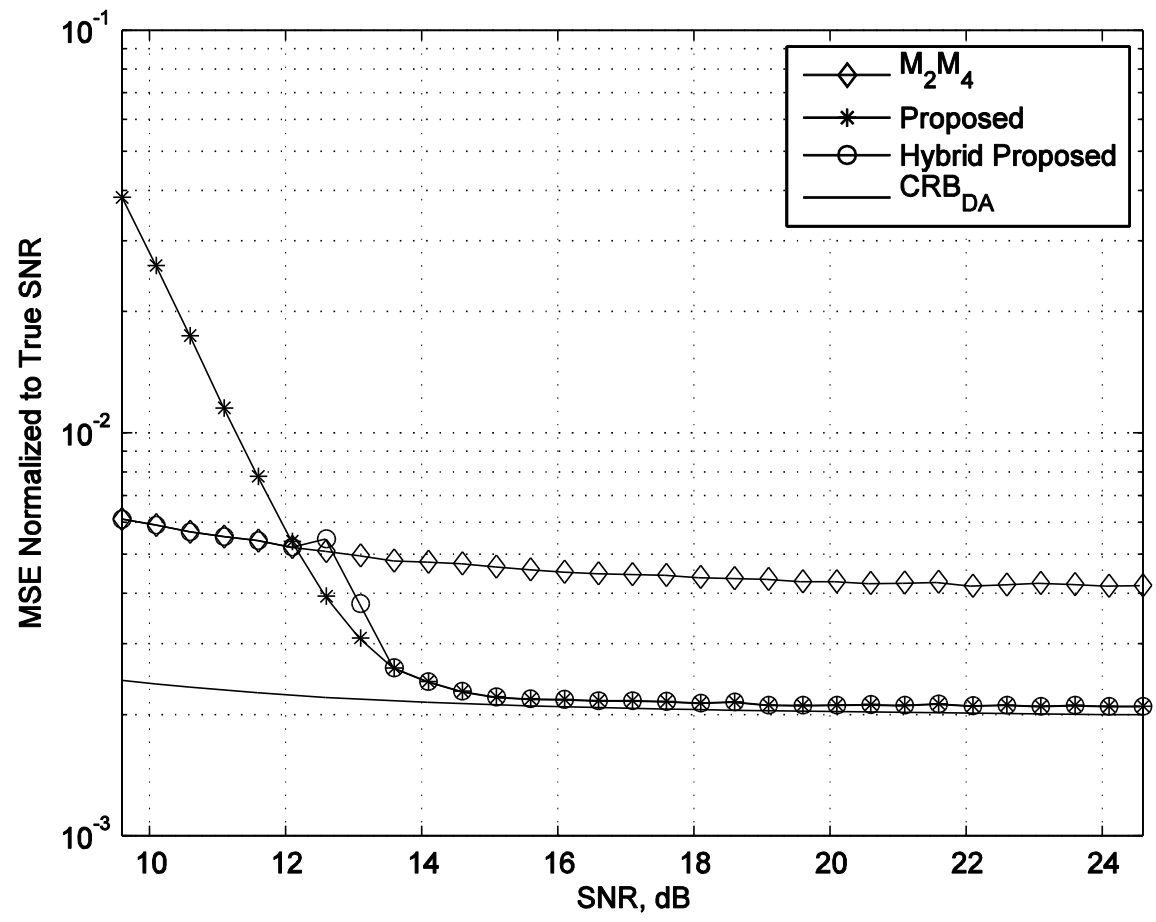


Table 1

\begin{tabular}{|l|l|l|l|}
\hline Operation & $\hat{S}_{D D M L}$ & $\hat{S}_{\text {proposed }}$ & $\hat{S}_{M_{2} M_{4}}$ \\
\hline Real Multiplications & $2 L+2$ & 11 & $3 L+4$ \\
\hline Real Additions & $2 L-1$ & $1.5 L-1$ & $L$ \\
\hline
\end{tabular}

